\title{
Enhancement of noncontact friction between closely spaced bodies by two-dimensional systems
}

\author{
A. I. Volokitin, ${ }^{1,2}$ B. N. J. Persson, ${ }^{1}$ and H. Ueba ${ }^{3}$ \\ ${ }^{1}$ Institut für Festkörperforschung, Forschungszentrum, Jülich, D-52425, Germany \\ ${ }^{2}$ Samara State Technical University, 443100 Samara, Russia \\ ${ }^{3}$ Department of Electronics, Toyama University, Gofuku, Toyama, 930-8555, Japan \\ (Received 29 July 2005; revised manuscript received 6 December 2005; published 26 April 2006)
}

\begin{abstract}
We consider the effect of an external bias voltage and the spatial variation of the surface potential on the damping of cantilever vibrations. The electrostatic friction is due to energy losses in the sample created by the electromagnetic field from the oscillating charges induced on the surface of the tip by the bias voltage and spatial variation of the surface potential. A similar effect arises when the tip is oscillating in the electrostatic field created by charged defects in a dielectric substrate. The electrostatic friction is compared with the van der Waals friction originating from the fluctuating electromagnetic field due to quantum and thermal fluctuation of the current density inside the bodies. We show that the electrostatic and van der Waals friction can be greatly enhanced if on the surfaces of the sample and the tip there are two-dimensional (2D) systems, e.g., a 2D electron system or incommensurate layers of adsorbed ions exhibiting acoustic vibrations. We show that the damping of the cantilever vibrations due to the electrostatic friction may be of similar magnitude as the damping observed in recent experiments by Stipe et al. [Phys. Rev. Lett. 87, 096801 (2001)]. We also show that at short separation the van der Waals friction may be large enough to be measured experimentally.
\end{abstract}

DOI: 10.1103/PhysRevB.73.165423

PACS number(s): 68.35.Af, 68.37.Ps, 73.20.Mf

\section{INTRODUCTION}

A great deal of attention has been devoted to non-contact friction between an atomic force microscope tip and a substrate. ${ }^{1-5}$ This problem is related to the role of noncontact friction for ultrasensitive force detection experiments. The ability to detect small forces is inextricably linked to friction via the fluctuation-dissipation theorem. According to this theorem, the random force that makes a small particle jitter would also cause friction if the particle were dragged through the medium. For example, the detection of single spins by magnetic resonance force microscopy ${ }^{6}$ which has been proposed for three-dimensional atomic imaging ${ }^{7}$ and quantum computation, ${ }^{8}$ will require force fluctuations (and consequently the friction) to be reduced to unprecedented levels. In addition, the search for quantum gravitation effects at short length scale, ${ }^{9}$ and future measurements of the dynamical Casimir forces,${ }^{10}$ may eventually be limited by noncontact friction effects. Noncontact friction is also responsible for the frictional drag force between twodimensional (2D) quantum wells. ${ }^{11-13}$

In noncontact friction the bodies are separated by a potential barrier thick enough to prevent electrons or other particles with a finite rest mass from tunneling across it, but allowing interaction via the long-range electromagnetic field, which is always present in the gap between bodies and can have different origin. The presence of an inhomogeneous tipsample electric field is difficult to avoid, even under the best experimental conditions. ${ }^{3}$ For example, even if both the tip and the sample were metallic single crystals, the tip would still have corners, and more than one crystallographic plane exposed. The presence of atomic steps, adsorbates, and other defects will also contribute to the spatial variation of the surface potential. This is referred to as "patch effect." The surface potential can also be easily changed by applying a voltage between the tip and the sample. An inhomogeneous electric field can also be created by charged defects embedded in a dielectric sample. The relative motion of the charged bodies will produce friction, which will be denoted as the electrostatic friction.

The electromagnetic field can also be created by the fluctuating current density, due to thermal and quantum fluctuations inside the solids. This fluctuating electromagnetic field gives rise to the well-known long-range attractive van der Waals interaction between two bodies, ${ }^{14}$ and is responsible for radiative heat transfer. If the bodies are in relative motion, the same fluctuating electromagnetic field will give rise to a friction which is frequently named as the van der Waals friction.

Recently Stipe et al. ${ }^{3}$ observed noncontact friction between a gold surface and a gold-coated cantilever as a function of tip-sample spacing $d$, temperature $T$, and bias voltage $V$. The friction force $F$ acting on the tip was found to be proportional to the velocity $v, F=\Gamma v$. For vibration of the tip parallel to the surface they found $\Gamma(d)=\alpha(T)\left(V^{2}+V_{0}^{2}\right) / d^{n}$, where $n=1.3 \pm 0.2$ and $V_{0} \sim 0.2 \mathrm{~V}$. At $295 \mathrm{~K}$, for the spacing $d=100 \AA$ they found $\Gamma=1.5 \times 10^{-13} \mathrm{kgs}^{-1}$. An applied voltage of $1 \mathrm{~V}$ resulted in a friction $\Gamma=3 \times 10^{-12} \mathrm{~kg} / \mathrm{s}$ at $300 \mathrm{~K}$ with $d=20 \mathrm{~nm}$.

In Ref. 3 the noncontact friction has also measured for fused silica samples. Near the silica surface the friction was found to be an order of magnitude larger than for the gold sample. The silica sample had been irradiated with $\gamma$ rays which produce $E^{\prime}$ centers (Si dangling bonds) at a density of $7 \times 10^{17} \mathrm{~cm}^{-3}$. Although the sample is electrically neutral overall, the $E^{\prime}$ centers are known to be positively charged, creating enhanced field inhomogeneity and causing the noncontact friction to rise another order of magnitude.

Attempts to explain the observed friction in terms of the van der Waals friction have not met with much success since the van der Waals friction for good conductors like copper 
has been shown ${ }^{15-17}$ to be many orders of magnitude smaller than the friction observed by Stipe et al.. In Ref. 18 it was proposed that the van der Waals friction may be strongly enhanced between a high-resistivity mica substrate and silica tip. However, in Ref. 3 the mica substrate and silica tip were coated by gold films thick enough to completely screen the electrodynamic interaction between the underlying dielectrics.

At small separation $d \sim 1 \mathrm{~nm}$, resonant photon tunneling between adsorbate vibrational modes on the tip and the sample may increase the friction by seven orders of magnitude in comparison with the good conductors surfaces. ${ }^{19,20}$ However, the distance dependence $\left(\sim 1 / d^{6}\right)$ is stronger than observed experimentally. ${ }^{3}$

Recently, a theory of noncontact friction was suggested where the friction arises from ohmic losses associated with the electromagnetic field created by moving charges induced by the bias voltage.$^{21}$ In the case of a spherical tip this theory predicts the same weak distance dependence of the friction as observed in the experiment, but the magnitude of the friction is many orders of magnitude smaller than found experimentally. However, we have shown that the electrostatic friction can be greatly enhanced if there is an incommensurate adsorbed layer exhibiting acoustic vibrations. ${ }^{22}$ This theory gives an explanation for the experimentally observed bias voltage contribution to the noncontact friction.

In this article we extend the theory presented in Ref. 22 to include the contribution to friction from the spatial variation of the surface potential and from the spatial fluctuation of the electric charge of charged defects in the bulk of the dielectric. We also show that the electrostatic friction as well as the van der Waals friction can be greatly enhanced for 2D systems, e.g., a 2D electron system or an incommensurate layer of adsorbed ions exhibiting acoustic vibrations. The origin of this enhancement is related to the fact that the screening in 2D systems is much less effective than for 3D systems. An atomic force microscope tip charged by the bias voltage, or by the spatial variation of the surface potential, and moving close to the metal surface will induce "image" charge in the 2D system. Because of the finite response time this "image" charge will lag behind the tip, and this effect results in force acting on the tip, referred to as the "electrostatic friction." However, the weaker screening effect in the 2D system will result in a much weaker restoring force, which occurs when the "image charge" is displaced from the equilibrium position, and this results in larger lag of the "image" charge in 2D systems in comparison with 3D systems.

Another contribution to the friction from the electric field is associated with the time-dependent stress acting on the surface due to the tip oscillations. This stress can excite acoustic phonons or induce nonadiabatic time-dependent deformation. In this paper we develop theories of phonon and internal friction due to the time-dependent stress acting on the surface. We show that this stress depends on the bias voltage as $V^{2}$, resulting in to the friction coefficient $\Gamma \sim V^{4}$. Thus this mechanism can be ruled out as an explanation of the experimental data observed in Ref. 3 , where $\Gamma \sim V^{2}$, In the case of phonon friction only phonons with $q<\omega / c_{s}$ can be excited, where $q$ is the component of the wave vector parallel to the surface of the substrate, $\omega$ is the frequency of

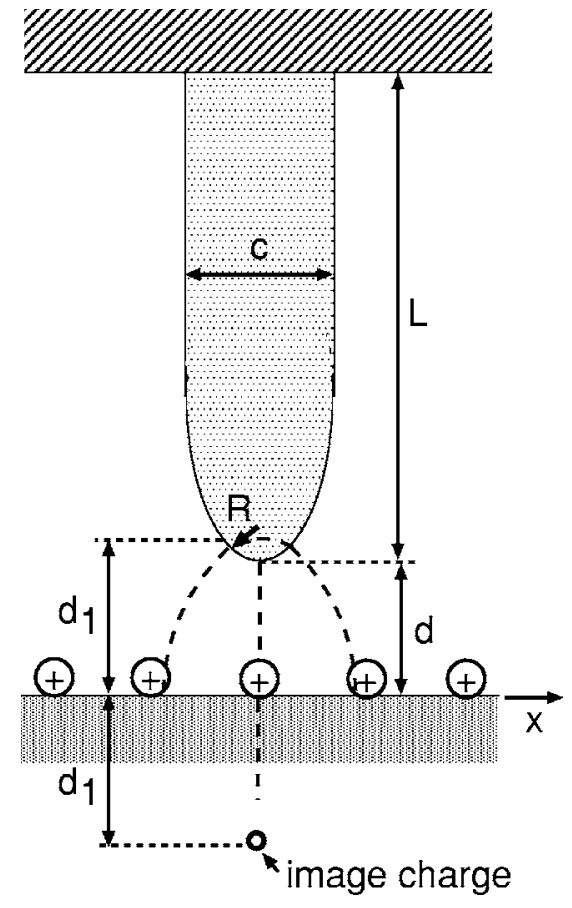

FIG. 1. Scheme of the tip-sample system. The tip shape is characterized by its length $L$ and the tip radius of curvature $R$.

the tip oscillations, and $c_{s}$ is the sound velocity. Thus in the phase space the area occupied by the excited phonons $\sim\left(\omega / c_{s}\right)^{2}$. For electromagnetic mechanisms of the friction (which include the electrostatic and van der Waals friction) all components of the electromagnetic field with $q<d_{1}^{-1}$, where $d_{1}$ is the radius of interaction (see below, typically $d_{1} \sim 100 \mathrm{~nm}$ ), are important. Thus for the metal substrate in the typical case $\left(\omega d_{1} / c_{s}\right)^{2} \ll 1$, the phonon friction is negligible in the comparison with the electromagnetic friction.

\section{ELECTROSTATIC FRICTION DUE TO A BIAS VOLTAGE AND THE SPATIAL VARIATION OF THE SURFACE POTENTIAL}

\section{A. A general theory}

We begin by considering a model in which the tip of a metallic cantilever of length $L$ is a section of a cylindrical surface with the radius of curvature $R$ (Fig. 1). The cantilever is perpendicular to a flat sample surface, which occupies the $x y$ plane, with the $z$ axis pointing outside the sample. The tip displacement $\mathbf{u}(t)=\hat{x} u_{0} e^{-i \omega t}$ is assumed to be parallel to the surface (along the $x$ axis), which will be a good approximation when the oscillation amplitudes $u_{0}$ is sufficiently small. The cantilever width $w$, i.e., the size in the direction perpendicular to the $x z$ plane, is taken to be much larger than the thickness $c(w \gg c)$, and $d$ is the separation between the tip and the sample surface. It is straightforward to obtain the static electric field distribution in the practically important case of small distances $d$ such that the electrostatic field of the entire cylinder is effectively the same as that due to its bottom part. (The criterion that $d$ is satisfied for this to be the case is given by $\sqrt{d / R} \ll 1$.) The problem is then reduced to 
solving the two-dimensional Laplace equation with the boundary conditions that the potential has constant values $V$ and 0 at the metallic surfaces of the tip and the substrate. The electric field distribution outside the conductors is equal to the field due to two charged wires passing through points at $z= \pm d_{1}= \pm \sqrt{(d+R)^{2}-R^{2} .}{ }^{23}$ The wires have charges $\pm Q$ per unit length $Q=C V$, where $C^{-1}=2 \ln \left[\left(d+R+d_{1}\right) / R\right]$. The electric potential at a point $\mathbf{r}$ exterior to the tip and sample is given by

$$
\begin{aligned}
\varphi_{0}(\mathbf{r}) & =-2 Q\left[\ln \left|\mathbf{r}-\mathbf{r}_{+}\right|-\ln \left|\mathbf{r}-\mathbf{r}_{-}\right|\right] \\
& =Q \int_{-\infty}^{\infty} \frac{d q}{|q|} \mathbf{e}^{i q x}\left[e^{-|q|\left|z-z_{+}\right|}-e^{-|q|\left|z-z_{-}\right|}\right],
\end{aligned}
$$

where $\mathbf{r}_{ \pm}= \pm \hat{z} d_{1}$. The attractive cantilever-surface force can be calculated straightforwardly using Eq. (1). ${ }^{21}$

A somewhat different picture applies in the case of an oscillating charged tip. The cantilever charge is not changed when its tip moves parallel to the surface, while the sample charge varies in time at any fixed point. The electric field from the oscillating tip will induce an electric charge in the sample and this will result in an induced electric field outside the sample. The oscillating electric potential due to the tip oscillation at a point $\mathbf{r}$ exterior to the tip and sample is given by

$$
\varphi_{1}(\mathbf{r}, \mathbf{t})=\varphi_{1}(\mathbf{r}) e^{-i \omega t}+\text { c.c. },
$$

where

$$
\varphi_{1}(\mathbf{r})=i Q u_{0} \int_{-\infty}^{\infty} \frac{d q q}{|q|} e^{i q x}\left[e^{-|q|\left|z-z_{+}\right|}-e^{-|q|\left|z-z_{-}\right|} R_{p}(q, \omega)\right],
$$

and $R_{p}(q, \omega)$ is the reflection amplitude for the $p$-polarized electromagnetic waves. The electric field is given by $\mathbf{E}(\mathbf{r})=$ $-\nabla \varphi(\mathbf{r})$. The energy dissipation per unit time induced by the electromagnetic field inside of the metallic substrate is determined by integrating the Poynting vector over the surface of the metal and is given by

$$
\begin{aligned}
P & =\frac{c}{4 \pi} \int d S \hat{z} \cdot\left[\mathbf{E}(\mathbf{r}) \times \mathbf{B}^{*}(\mathbf{r})\right]_{z=+0}+\text { c.c. } \\
& =-\frac{i \omega}{4 \pi} \int d S\left(\varphi_{1}(\mathbf{r}) \frac{d}{d z} \varphi_{1}^{*}(\mathbf{r})\right)_{z=+0}+\text { c.c. } \\
& =4 \omega Q^{2}\left|u_{0}\right|^{2} w \int_{0}^{\infty} d q q e^{-2 q d_{1}} \operatorname{Im} R_{p}(\omega, q) .
\end{aligned}
$$

Taking into account that the energy dissipation per unit time must be equal to $2 \omega^{2} \Gamma\left|u_{0}\right|^{2}$, using (4) gives the friction coefficient:

$$
\Gamma=\lim _{\omega \rightarrow 0} 2 C^{2} V^{2} w \int_{0}^{\infty} d q q e^{-2 q d_{1}} \frac{\operatorname{Im} R_{p}(\omega, q)}{\omega} .
$$

Without derivation Eq. (5) was first presented in Ref. 22. Now we assume that the electric potential on the surface of the tip is inhomogeneous, consisting of the domains or "patches." Thus the cylinder with linear size $w$ is divided into smaller cylinders with the linear size $w_{i}\left(w=\sum_{i} w_{i} \gg w_{i}\right.$ $\gg \sqrt{d R}$ ), and with the surface potential $V_{i s}=V+V_{i}$, where $V$ is the bias voltage and $V_{i}$ is the randomly fluctuating surface potential for the domain $i$. In the case of a cylindrical tip geometry all domains give independent contribution to friction which can be obtained from Eq. (5) after replacement $V \rightarrow V+V_{i}$ and $w \rightarrow w_{i}$. The contribution to friction from all domains is given by

$$
\begin{aligned}
\Gamma & =\sum_{i} \Gamma_{i}=\sum_{i} \lim _{\omega \rightarrow 0} 2 C^{2}\left(V+V_{i}\right)^{2} w_{i} \int_{0}^{\infty} d q q e^{-2 q d_{1}} \frac{\operatorname{Im} R_{p}(\omega, q)}{\omega} \\
& =\lim _{\omega \rightarrow 0} 2 C^{2}\left(V^{2}+V_{0}^{2}\right) w \int_{0}^{\infty} d q q e^{-2 q d_{1}} \frac{\operatorname{Im} R_{p}(\omega, q)}{\omega}
\end{aligned}
$$

where we take into account that the average value of the fluctuating surface potential $\left\langle V_{i}\right\rangle=\Sigma_{i} w_{i} V_{i} / w=0$ and $V_{0}^{2}$ $=\Sigma_{i} w_{i} V_{i}^{2} / w$, so that $V_{0}$ is the root mean square variation of the surface potential. According to Eq. (6), bias voltage and patch contributions to the friction have the same dependence on $d$. Sukenik et al., studied the root mean square variation of the surface potential due to thermally evaporated gold using the Stark effect in sodium atoms. ${ }^{24}$ The films were partially optically transparent with a thickness of $42 \mathrm{~nm}$ and heated at $120^{\circ} \mathrm{C}$ for several hours in vacuum. They deduced the magnitude of the fluctuating surface potential to be $V_{0}$ $=0.15 \mathrm{~V}$ and showed that the scale of the lateral variation of the surface potential is of the order of the film thickness. The measurement of the noncontact friction between a gold tip and the gold sample gave $V_{0} \sim 0.2 \mathrm{~V}$ (Ref. 3), thus confirming the prediction of the theory that this parameter is determined by the root mean square variation of the surface potential.

Now, let us consider the spherical tip (radius $R$ ) with the constant voltage surface domains with the linear size $R_{i}$. If $R \gg R_{i} \gg \sqrt{d R}$, the domain on the apex of the tip will give the main contribution to the friction. In this case we can neglect the spatial variation of the surface potential, and the electric field induced by the bias voltage is approximately the same as that which would be produced in the vacuum region between two point charges $\pm Q_{i}= \pm C\left(V+V_{i}\right)$ located at

$$
z= \pm d_{1}= \pm \sqrt{3 R d / 2+\sqrt{(3 R d / 2)^{2}+R d^{3}+d^{4}}}
$$

where

$$
C=\frac{d_{1}^{2}-d^{2}}{2 d}
$$

It can been shown that the electrostatic force between the tip and the metal surface within this approximation agrees very well with the exact expression for a sphere above a metal surface. ${ }^{25}$ The vibrations of the tip will produce an oscillating electromagnetic field, which in the vacuum region coincides with the electromagnetic field of an oscillating point charge. The friction coefficient for a point charge moving parallel to the surface due to the electromagnetic energy losses inside the sample is determined by ${ }^{26}$ 


$$
\Gamma_{\|}=\lim _{\omega \rightarrow 0} \frac{Q_{i}^{2}}{2} \int_{0}^{\infty} d q q^{2} e^{-2 q d_{1}} \frac{\operatorname{Im} R_{p}(\omega, q)}{\omega} .
$$

For motion normal to the surface, $\Gamma_{\perp}=2 \Gamma_{\|}$. Thus, just as for the cylindrical tip geometry, for a spherical tip the friction depends quadratically on the bias voltage. However, for a spherical tip the parabola begins from zero in contrast to a cylindrical tip, where the parabola begins from a finite positive value.

\section{B. Clean surface}

For a clean flat surface in the electrostatic limit $(q$ $\gg \omega / c_{0}$, where $c_{0}$ is the light velocity) the reflection coefficient is determined by the well-known Fresnel formula

$$
R_{p}=\frac{\epsilon-1}{\epsilon+1} .
$$

In this case, for the tip radius $R \gg d$ and for a metal with the dielectric function $\epsilon=1+4 \pi i \sigma / \omega$, where $\sigma$ is the conductivity, Eq. (6) gives

$$
\Gamma_{c l}^{c}=\frac{w\left(V^{2}+V_{0}^{2}\right)}{2^{6} \pi \sigma d^{2}} .
$$

Neglecting the contribution from the spatial variation of the surface potential, this formula was obtained recently in Ref. 21 using a different approach. With $w=7 \times 10^{-6} \mathrm{~m}$ and $\sigma$ $=4 \times 10^{17} \mathrm{~s}^{-1}$ ( corresponds to gold at $300 \mathrm{~K}$ ), and with $d$ $=20 \mathrm{~nm}$ and $V=1 \mathrm{~V}$, Eq. (11) gives $\Gamma=2.4 \times 10^{-20} \mathrm{~kg} / \mathrm{s}$, which is eight orders of magnitude smaller than the experimental value $3 \times 10^{-12} \mathrm{~kg} / \mathrm{s}^{3}$

Assuming $R \gg d$, using (9) and (10) gives the friction between a spherical tip and a clean sample surface:

$$
\Gamma_{c l}^{s}=\frac{3^{1 / 2} R^{1 / 2} V^{2}}{2^{7} d^{3 / 2} \pi \sigma} .
$$

This expression is only a factor 1.6 smaller that the result obtained independently in Ref. 21 using a less general approach. For the same parameters as above and at $d=20 \mathrm{~nm}$, the friction for a spherical tip is two orders of magnitude smaller than for the cylindrical tip.

To get insight into possible mechanisms of the enhancement of noncontact friction it is instructive to note that qualitatively Eq. (11) can be obtained from the following simple geometrical arguments. ${ }^{27}$ The vibrating tip will induce current in the sample in a volume with the spatial dimensions $L_{x}, L_{y}$, and $L_{z}$. The instantaneous dissipated power in the sample is given by $P \sim I^{2} r$, where $I$ is the current and $r$ is the effective resistivity. The current $I$ is proportional to the tip velocity $v_{x}$ and can be written as $I \sim v_{x} Q_{t} / L_{x}$, where $Q_{t}$ is the charge of the tip. The effective resistance $r$ can be approximated by the macroscopic relation $r=\rho L_{x} / L_{y} L_{z}$, where $\rho$ is the resistivity. Using these simple expressions for current $I$ and resistance, and using the relation $Q_{t}=C_{t} V_{s}$ (where $C_{t}$ is the tip-sample capacitance) for the induced charge, the instantaneous power dissipation is

$$
P=I^{2} r \sim \rho \frac{v_{x}^{2} C_{t}^{2} V_{s}^{2}}{L_{x} L_{y} L_{z}} .
$$

Comparing this expression with $P=\Gamma v_{x}^{2}$ we get

$$
\Gamma \sim \rho \frac{C_{t}^{2} V_{s}^{2}}{L_{x} L_{y} L_{z}} .
$$

For a cylindrical tip vibrating above the clean surface, $L_{y}$ $\sim w$ and $L_{x} \sim L_{z} \sim d_{1}$. For $d \ll R$ the tip-sample capacitance $C_{t} \sim w \sqrt{R / 8 d}$ and $d_{1} \sim \sqrt{2 d R}$. Substituting these expressions in Eq. (14) gives Eq. (11) to within a numerical factor of order of unity. From Eq. (14) it follows that the friction will increase when the thickness $L_{z}$ of the "dissipation volume" decreases. This is the reason why $2 \mathrm{D}$ systems may exhibit higher friction than 3D systems.

\section{Film on top of a high-resistivity substrate}

From the qualitative arguments given above it follows that for a thin metal film on top of a high resistivity substrate, e.g., a dielectric or a high resistivity metal, the friction will be larger than for a semi-infinite sample with clean surface and with the same bulk conductivity as for a film . In this case the thickness $L_{z}$ of the volume, where the dissipation occurs, will be determined by the thickness of the film, and according to Eq. (14) this will give rise to a strong enhancement of the friction.

For a planar film with thickness $d_{f}$ and dielectric constant $\epsilon_{2}$ on top of a substrate with dielectric constant $\epsilon_{3}$, the reflection coefficient is determined by

$$
R_{p}=\frac{R_{p 21}-R_{p 23} \exp \left(-2 q d_{f}\right)}{1-R_{p 21} R_{p 23} \exp \left(-2 q d_{f}\right)},
$$

where

$$
R_{p i j}=\frac{\epsilon_{i}-\epsilon_{j}}{\epsilon_{i}+\epsilon_{j}},
$$

where index 1 is associated with vacuum. For a metallic film on a dielectric substrate, or a metallic film on a metallic substrate with $\sigma_{2} \gg \sigma_{3}$, for $d_{1} \gg d_{f}$ and $R \gg d$ Eqs. (6) and (15) give

$$
\Gamma_{f}^{c}=\frac{w\left(V^{2}+V_{0}^{2} R\right)^{1 / 2}}{2^{9 / 2} \pi \sigma_{2} d_{f} d^{3 / 2}} .
$$

This is greater by a factor of $2 d_{1} / d_{f}$ than the corresponding friction for the infinitely thick sample. For a thin film the effective resistivity of the substrate is increased, giving rise to additional ohmic dissipation. In Ref. 21, Eq. (17) was obtained for sample with finite thickness.

\section{2D system on top of a dielectric or metal substrate}

Let us now consider a 2D system, e.g., electronic surface states or a quantum well, or an incommensurate layer of ions adsorbed on a metal surface. For example, for the $\mathrm{Cs} / \mathrm{Cu}(100)$ system experiment suggests the existence of an acoustic film mode, even for the very dilute phase $(\theta \approx 0.1)$. This implies that the $\mathrm{Cs} / \mathrm{Cu}(100)$ adsorbate layer experi- 
ences a negligible surface pinning potential. The reflection coefficient for $p$-polarized electromagnetic waves can be obtained using the approach proposed in Ref. 28. This gives (see Ref. 22, detailed derivation is given in Appendix A)

$$
R_{p}=\frac{1-1 / \epsilon+4 \pi q n_{a} \alpha_{\|} / \epsilon-q a\left(1-4 \pi n_{a} q \alpha_{\|}\right)}{1+1 / \epsilon+4 \pi q n_{a} \alpha_{\|} / \epsilon+q a\left(1+4 \pi n_{a} q \alpha_{\|}\right)},
$$

where $n_{a}$ is the concentration of the free carries of the charge per unit area. The polarizability $\alpha_{\|}$for the $2 \mathrm{D}$ system in the direction parallel to the surface is taken to be

$$
\alpha_{\|}=-\frac{e^{* 2}}{M\left(\omega^{2}+i \omega \eta\right)},
$$

where $\eta$ is the damping constant, and $e^{*}$ and $M$ are the effective charge and the mass of the moving particles, respectively. In comparison with the expression obtained in Ref. 28 , Eq. (18) takes into account that the 2D system is located a distance $a$ away from the image plane of the metal. Although this correction to the reflection coefficient is of order $q a \ll 1$, for a $2 \mathrm{D}$ system on top of a good conductors $(|\epsilon|$ $\gg 1$ ), it gives the most important contribution to the energy dissipation.

For good metals $(|\epsilon| \gg 1)$, from Eq. (18) we get

$$
\operatorname{Im} R \approx \frac{2 \omega \eta q a \omega_{q}^{2}}{\left(\omega^{2}-\omega_{q}^{2}\right)^{2}+\omega^{2} \eta^{2}},
$$

where $\omega_{q}^{2}=4 \pi n_{a} e^{* 2} a q^{2} / M$. In the case of a $2 \mathrm{D}$ structure on top of a dielectric, the factor qa in Eq. (20) and in the expression for $\omega_{q}^{2}$ must be replaced by $1 / \epsilon$, where $\epsilon$ is the dielectric function of the substrate. Using (20) in (6) for $R$ $\gg d$ we get

$$
\Gamma_{a d}^{c}=\frac{w \eta M R^{1 / 2}\left(V^{2}+V_{0}^{2}\right)}{2^{9 / 2} d^{3 / 2} \pi n_{a} e^{* 2}} .
$$

This friction exhibits the same distance dependence as observed experimentally. ${ }^{3}$ The same expression for the friction is valid for a $2 \mathrm{D}$ structure on top of a dielectric. Comparing Eqs. (11) and (21) we find that a 2D structure on top of a substrate gives the same friction as for a semi-infinite sample with the clean surface and with the effective bulk conductivity $\sigma_{e f f}=n_{a} e^{* 2} / M \eta 2 d_{1}$. We obtain agreement with experiment at $d=20 \mathrm{~nm}$ if $\sigma_{\text {eff }} \approx 4 \times 10^{9} \mathrm{~s}^{-1}$. In the case of a $2 \mathrm{D}$ electron system, for $R=1 \mu \mathrm{m}$ such an effective conductivity is obtained if $\eta=10^{14} \mathrm{~s}^{-1}$ and $n_{a}=10^{15} \mathrm{~m}^{-2}$. For Cs $/ \mathrm{Cu}(100)$, for $n_{a}=10^{18} \mathrm{~m}^{-2}(\theta \approx 0.1)$ the electric charge of the Cs ions $e^{*}=0.28 e .{ }^{29}$ Due to the similarities of $\mathrm{Cu}$ and Au surfaces, a similar effective charge can be expected for the Cs/Au surface. For such a 2D system agreement with experiment is obtained for $n_{a}=10^{18} \mathrm{~m}^{-2}$ and $\eta=10^{11} \mathrm{~s}^{-1}$. In Ref. 22 we estimated the damping parameter for a $\mathrm{Cs}$ atom associated with the covalent bond $\eta_{\| \text {cov }}=3 \times 10^{9} \mathrm{~s}^{-1}$. ${ }^{22}$ However, the collisions between the ions, and between the ions and other surface defects, will also contribute to $\eta$. In this case $\eta_{c o l}$ $\sim v_{T} / l$ where $v_{T} \sim \sqrt{k_{B} T / M}$, and $l$ is the ion mean free path. For $T=293 \mathrm{~K}$ and $l \sim 1 \mathrm{~nm}$ we get $\eta_{c o l}=10^{11} \mathrm{~s}^{-1}$.
For a spherical tip, with a 2D system on top of the substrate, from Eqs. (20) and (9) for $R \gg d$ we get the contribution to the friction from the $2 \mathrm{D}$ system:

$$
\Gamma_{a d}^{s}=\frac{3 R M \eta V^{2}}{2^{6} d \pi n_{a} e^{* 2}} .
$$

At $d=20 \mathrm{~nm}$ this friction is approximately two order of magnitude smaller than for the cylindrical tip.

\section{E. Friction due to spatial fluctuations of static charge in the bulk of the sample}

In this section we consider a dielectric substrate with a stationary, inhomogeneous distribution of charged defects. Such a situation was investigated experimentally ${ }^{3}$ by employing a fused silica sample irradiated with $\gamma$ rays. In the course of irradiation, positively charged centers (Si dangling bonds) are generated. Randomly distributed positive charges are compensated by randomly distributed negative charges, thus on average the sample is electrically neutral. We model the sample as consisting of microscopically small volume elements $\Delta V_{i}$. Each element is chosen sufficiently small that not more than one charge center is present in it. The electric charge $q_{i}$ of each element is equal to $\pm e$ or 0 , in such away that the average $\left\langle q_{i}\right\rangle=0$. We will consider the fluctuations of charges in different volume elements $i, j$ to be statistically independent, so that $\left\langle q_{i} q_{j}\right\rangle=0$ for $i \neq j$. The mean square of charge fluctuations within a given element $\left\langle q_{i} q_{i}\right\rangle \approx 2 N e^{2}$, where $N$ is the average number of positive charges in one volume element. In the absence of the cross terms, the average tip-sample friction coefficient is determined by adding friction coefficients from all charges $q_{i}$. According to Eq. (9), the contribution to the friction coefficient from charge $q_{i}$ in the element $\Delta V_{i}$ is given by

$$
\Delta \Gamma_{i}=\lim _{\omega \rightarrow 0} N e^{2} \int_{0}^{\infty} d q q^{2} e^{-2 q d_{i}} \frac{\operatorname{Im} R_{p}(\omega, q)}{\omega},
$$

where $d_{i}=D\left(x_{i}, y_{i}\right)-z_{i}$. Here the coordinates $x_{i}, y_{i}, z_{i}$ give the position of the $i$ th volume element in the substrate, and $D\left(x_{i}, y_{i}\right)$ is the distance between the substrate and points $x_{i}, y_{i}$ located on the surfaces of the tip. The total friction coefficient is obtained by summing over all the elements. Replacing the sum by an integral $\left(N \Sigma \rightarrow n \int d^{3} r\right.$, where $c$ is the number of the positive charge centers per unit volume), and integration over $z$ gives

$$
\Gamma=\lim _{\omega \rightarrow 0} \frac{n e^{2}}{2} \int_{0}^{\infty} d q q \int d x \int d y e^{-2 q D(x, y)} \frac{\operatorname{Im} R_{p}(\omega, q)}{\omega} .
$$

For a cylindrical tip $D(x, y)=d+x^{2} / 2 R$, and we get

$$
\Gamma_{\|}^{c}=\lim _{\omega \rightarrow 0} \frac{\sqrt{\pi R} n e^{2} w}{2} \int_{0}^{\infty} d q q^{1 / 2} e^{-2 q d} \frac{\operatorname{Im} R_{p}(\omega, q)}{\omega} .
$$

Using the same parameters as in Sec. II B, for a gold tip separated by $d=10 \mathrm{~nm}$ from a dielectric sample with $n=7$ $\times 10^{17} \mathrm{~cm}^{-3}$ we get $\Gamma_{\|}=4.4 \times 10^{-20} \mathrm{~kg} \mathrm{~s}^{-1}$. 
For the tip surface with a $2 \mathrm{D}$ structure on it, using Eq. (20) we get

$$
\Gamma_{2 D}^{c}=\frac{1}{2^{5 / 2}}\left(\frac{e}{e^{*}}\right)^{2} \sqrt{\frac{R}{d}} \frac{n w}{n_{a}} M \eta=\frac{e^{2} n w}{16 \sigma_{e f f} d} .
$$

With $\sigma_{e f f}=n_{a} e^{* 2} / 2 M \eta d_{1}=4 \times 10^{9}, \quad n=7 \times 10^{17} \mathrm{~cm}^{-3}$, and with the other parameters the same as before, we get for $d$ $=10 \mathrm{~nm}, \Gamma_{2 D \|}^{c}=3.5 \times 10^{-12} \mathrm{~kg} \mathrm{~s}^{-1}$, which is nearly the same as was observed experimentally. ${ }^{3}$ Thus our theory of friction between a gold tip and silica substrate with an inhomogeneous distribution of the charged defects is consistent with the theory of friction between a gold tip and gold substrate (see Sec. II D). In both theories we have assumed that the gold surfaces are covered by a 2D structure.

The study above has ignored the screening of the electric field in the dielectric substrate. This can be justified in the case of very small tip-sample separations (substantially smaller than screening length), as only defects in the surface layer of thickness $d$ contribute to the integral in Eq. (24). When the screening is important, the effective electric field outside the sample will be decreased by the factor $(\varepsilon+1) / 2,{ }^{23}$ and the friction coefficient will be decreased by the factor $[(\varepsilon+1) / 2]^{2}$, which is equal to $\approx 6.25$ in the case of silica. However, the inhomogeneity of the surface of the tip may be larger than that of the sample surface, so that the damping parameter $\eta$ may be larger for the 2D structure on the surface of the tip. This increase in $\eta$ and screening effects will compensate each other.

\section{VAN DER WAALS FRICTION}

In this section we consider the van der Waals friction between two surfaces covered by $2 \mathrm{D}$ systems. The frictional stress between two flat surfaces to linear order in the relative velocity $v$ can be written in the form $\sigma=\gamma v$. According to Ref. 15, in the case of the van der Waals friction the contribution to the friction coefficient $\gamma_{\|}$from the $p$-polarized electromagnetic waves is given by

$$
\begin{aligned}
\gamma= & \frac{\hbar}{2 \pi^{2}} \int_{0}^{\infty} d \omega\left(-\frac{\partial n}{\partial \omega}\right) \int_{0}^{\infty} d q q^{3} e^{-2 q d} \\
& \times \operatorname{Im} R_{1 p} \operatorname{Im} R_{2 p} \frac{1}{\left|1-e^{-2 q d} R_{1 p} R_{2 p}\right|^{2}},
\end{aligned}
$$

where $R_{1 p}$ and $R_{2 p}$ are the reflections coefficients for the surfaces, and $n=\left[\exp \left(\hbar \omega / k_{B} T\right)-1\right]^{-1}$. In Refs. 19 and 20 we have shown that resonant photon tunneling between two $\mathrm{Cu}(100)$ surfaces separated by $d=1 \mathrm{~nm}$ and covered by a low concentration of potassium atoms gives rise to a friction six orders of the magnitude larger than for clean surfaces. The adsorbate induced enhancement of the van der Waals friction is even larger for $\mathrm{Cs}$ adsorption on $\mathrm{Cu}(100)$. In this case, even at low Cs coverage $(\theta \sim 0.1)$, the adsorbed layer exhibits an acoustic branch for vibrations parallel to the surface, ${ }^{29}$ and, according to Eq. (18), at small frequencies the reflection coefficient is given by

$$
R_{p}=1-\frac{2 q a \omega_{q}^{2}}{\omega^{2}-\omega_{q}^{2}+i \omega \eta},
$$

where $\omega_{q}^{2}=4 \pi n_{a} e^{* 2} a q^{2} / M$. Using Eq. (28) in Eq. (27) for

$$
\frac{a}{\eta d} \sqrt{\frac{4 \pi n_{a} e^{* 2} a}{M d^{2}}} \ll 1
$$

gives

$$
\gamma \approx 0.62 \frac{k_{B} T a^{2}}{\eta d^{6}} .
$$

It is interesting to note that according to (29) $\gamma_{\|}$does not depend on $n_{a}, e^{*}$, and $M$. However, Eq. (28) is only valid when there are acoustic vibrations in the adsorbed layer. For Cs adsorbed on $\mathrm{Cu}(100)$ the acoustic vibrations exist only for $\theta \geqslant 0.1 .^{29}$ The friction coefficient for a cylindrical atomic force microscope tip can be estimated using 30,31

$$
\Gamma_{\|}^{c} \approx 2 w \int_{0}^{\infty} d x \gamma(z(x))=0.68 \frac{k_{B} T a^{2} R^{0.5} w}{\eta d^{5.5}},
$$

where $R$ is the radius of the curvature of the tip, $w$ is its width, and $\gamma_{\|}(z(x))$ is the friction coefficient between two flat surfaces at the separation $z(x)=d+x^{2} / 2 R$. In Sec. II we have shown that the experimental data in Ref. 3 can be explained by assuming that the gold surfaces are covered by an adsorbed layer of ions like $\mathrm{Cs}$ on $\mathrm{Cu}(100)$ with the damping constant $\eta \approx 10^{11} \mathrm{~s}^{-1}$. With this value of $\eta$ and using $a$ $=2.94 \AA,{ }^{29}, R=1 \mu \mathrm{m}, w=7 \mu \mathrm{m}$, and $T=293 \mathrm{~K}$ we find that if $d<3 \mathrm{~nm}$, the contribution from the van der Waals friction will dominate over the contribution from the electrostatic friction. However, in the experiment a strong enhancement in the friction was not observed at such short separation. Thus, most likely a 2D system of electronic origin is responsible for the enhancement of the electrostatic friction. In this case (see Sec. II) $\eta_{e l} \sim 10^{14} \mathrm{~s}^{-1}$ and the van der Waals friction will give a negligible contribution for practically all separations. Figure 2 shows how the friction between the copper tip and the copper substrate depends on the distance $d$, when the surfaces of the tip and the substrate are covered by a low concentration of the $\mathrm{Cs}$ atoms, and for clean surfaces. In comparison, the friction between two clean surfaces at the separation $d=1 \mathrm{~nm}$ is 11 orders of the magnitude smaller. However, the friction between clean surfaces shown in Fig. 2 was calculated in the local optic approximation. For parallel relative motion nonlocal optic effects are very important, ${ }^{20}$ and when it is taken into account, at $d=1 \mathrm{~nm}$ the friction between adsorbate covered surfaces will be seven orders of the magnitude larger than the friction between clean surfaces.

\section{PHONON AND INTERNAL NONCONTACT FRICTION}

\section{A. Noncontact friction due to excitation of substrate phonons}

Consider a tip which performs harmonic oscillation, $u$ $=u_{0} \exp (-\mathrm{i} \omega t)+$ c.c., above an elastic body with a flat surface. This will results in a fluctuating stress acting on the surface 


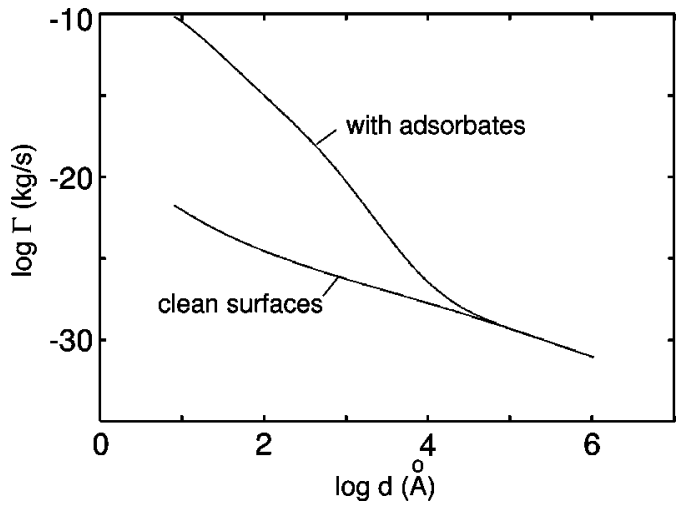

FIG. 2. The friction coefficient associated with the van der Waals friction between a copper tip and a copper substrate, both covered by low concentration of cesium atoms, as a function of the separation $d$. The cylindrical tip is characterized by the radius of curvature $R=1 \mu \mathrm{m}$ and the width $w=7 \mu \mathrm{m}$. The other parameters correspond to $\mathrm{Cs}$ adsorbed on $\mathrm{Cu}(100)$ at the concentration $n_{a}$ $=10^{18} \mathrm{~m}^{-2}$ (coverage $\left.\theta \approx 0.1\right):^{22,29} \quad e^{*}=0.28 e, \quad \eta=10^{11} \mathrm{~s}^{-1}, \quad a$ $=2.94 \AA, T=293 \mathrm{~K}$. (The base of the logarithm is 10 .)

of the solid which excite acoustic waves with parallel wave number $q<\omega / c_{s}$, where $c_{s}$ is the sound velocity. The stress $\sigma_{i z}$ acting on the surface of the elastic solid can be represented through the Fourier integral

$$
\sigma_{i z}(\mathbf{x}, t)=\int \frac{d^{2} q}{(2 \pi)^{2}} \sigma_{i}(\mathbf{q}) u_{0} e^{i \mathbf{q} \mathbf{x}-i \omega t}+\text { c.c. }
$$

Using the theory of elasticity (assuming an isotropic elastic medium for simplicity), one can calculate the displacement field $u_{i}$ on the surface $z=0$ in response to the surface stress distribution $\sigma_{i z}$ :

$$
u_{i}(\mathbf{x}, t)=\int \frac{d^{2} q}{(2 \pi)^{2}} M_{i j}(\mathbf{q}, \omega) \sigma_{j}(\mathbf{q}) u_{0} e^{i \mathbf{q} \mathbf{x}-i \omega t}+\text { c.c. }
$$

The energy dissipation per unit time equals

$$
\begin{aligned}
P & =\int d^{2} x\left\langle\dot{u}_{i}(\mathbf{x}, t) \sigma_{i z}(\mathbf{x}, t)\right\rangle \\
& =2 \omega \int \frac{d^{2} q}{(2 \pi)^{2}} \operatorname{Im} M_{i j}(\mathbf{q}, \omega) \sigma_{i}(\mathbf{q}) \sigma_{j}^{*}(\mathbf{q})\left|u_{0}\right|^{2},
\end{aligned}
$$

where $\langle\ldots\rangle$ stands for the time averaging. The explicit form of the stress tensor in the model of the elastic continuum is given in Ref. 32 (see also Appendix B). The energy dissipation per unit time must be equal to $\Gamma\left\langle\dot{u}(t)^{2}\right\rangle=\Gamma 2 \omega^{2}\left|u_{0}\right|^{2}$. Comparing of this expression with (33) gives

$$
\Gamma=\int \frac{d^{2} q}{(2 \pi)^{2}} \frac{\operatorname{Im} M_{i j}(\mathbf{q}, \omega)}{\omega} \sigma_{i}(\mathbf{q}) \sigma_{j}^{*}(\mathbf{q}) .
$$

At typical experimental conditions we have $\omega$ $\sim 10^{3}-10^{6} \mathrm{~s}^{-1}$ and $q r^{*}<\omega r^{*} / c_{s}<10^{-3} \ll 1$, where the effective radius of the interaction $r^{*} \approx \sqrt{d R}, d$ is the separation between the tip and the sample, and $R$ is the radius of curvature of the tip. In Appendix B it was shown that in this case the contribution to the friction from excitation of acous- tic waves can be determined by calculating the energy dissipation due to oscillating point force applied to the surface of the semi-infinite elastic continuum. These calculations were done in connection with the vibrational energy relaxation of adsorbates. ${ }^{33}$ According to this theory the friction coefficient for vibration of the tip normal to the surface is given by

$$
\Gamma_{\perp}=\frac{\xi_{\perp}}{4 \pi} \frac{K^{2}}{\rho c_{t}^{3}}
$$

where $\xi_{\perp} \approx 1.65, c_{t}$ is the transverse sound velocity of the solid, $\rho$ is the mass density of the sample, and $K=\partial F / \partial d$, where $F(d)$ is the static force acting on the tip due to interaction with the sample.

In Appendix B it was shown that for vibration of the tip parallel to the flat surface the friction coefficient due to excitation of the acoustic waves is given by

$$
\Gamma=\frac{\xi}{4 \pi} \frac{\omega^{2}}{\rho c_{t}^{5}} F_{z}^{2}(d)
$$

where $\xi_{\|} \approx 1.50$. From the comparison of Eqs. (35) and (36) we get that $\Gamma_{\|} / \Gamma_{\perp} \sim\left(\omega d / c_{t}\right)^{2} \ll 1$. We consider now two different contributions to the tip-sample interaction.

\section{Van der Waals interaction}

According to the Lifshitz theory, ${ }^{14}$ the stress $\sigma_{z z}(d)$ acting on the surface of two identical semi-infinite bodies due to van der Waals interaction at small separation $d \ll c / \omega_{p}$ (where $\omega_{p}$ is the plasma frequency) and $d \ll \lambda_{T}$ is given by

$$
\sigma_{z z}(d)=\frac{\hbar}{8 \pi^{2} d^{3}} \int_{0}^{\infty} d \xi \frac{[\varepsilon(i \xi)-1]^{2}}{[\varepsilon(i \xi)+1]^{2}} .
$$

In the Drude model the explicit form of $\varepsilon$ is

$$
\varepsilon(i \xi)=1+\frac{\omega_{p}^{2}}{\xi(\xi+\eta)} .
$$

For typical metal the damping constant $\eta \ll \omega_{p}$ and can be neglected when integrating Eq. (37). It follows from Eqs. (37) and (38) that

$$
\sigma_{z z}=\frac{\hbar \omega_{p}}{32 \sqrt{2} \pi d^{3}} .
$$

For the spherical tip of radius $R$ using the same approximation as in Eq. (30) we get

$$
F_{z}(d)=\frac{R \hbar \omega_{p}}{32 \sqrt{2} d^{2}}
$$

and

$$
K^{s}=\frac{R \hbar \omega_{p}}{16 \sqrt{2} d^{3}} .
$$

Similarly, in the case of a cylindrical tip we have

$$
F_{z}^{c}(d)=\frac{3 w R^{1 / 2} \hbar \omega_{p}}{2^{8} d^{5 / 2}}
$$

and 


$$
K^{c}=\frac{15 w R^{1 / 2} \hbar \omega_{p}}{2^{9} d^{7 / 2}} .
$$

For a copper tip separated from a copper substrate by $d$ $=10 \mathrm{~nm}$, and with $R=1 \mu \mathrm{m}, w=7 \mu \mathrm{m}$, we get for spherical tip $\Gamma^{s}=6.3 \times 10^{-18} \mathrm{~kg} \mathrm{~s}^{-1}$ and for cylindrical tip $\Gamma_{\perp}^{c}=1.3$ $\times 10^{-14} \mathrm{~kg} \mathrm{~s}^{-1}$. The phononic friction decreases as $d^{-6}$ and $d^{-7}$ for spherical and cylindrical tips, respectively.

\section{Electrostatic interaction due to a bias voltage}

In the presence of the bias voltage $V$ the attractive force between the tip and the sample at $d \ll R$ is given by

$$
F^{c}(d)=\frac{w V^{2} R^{1 / 2}}{2^{7 / 2} d^{3 / 2}}
$$

for a cylindrical tip and

$$
F^{s}(d)=\frac{R V^{2}}{4 d}
$$

for a spherical tip. For bias voltage $V=1$ Volt, and with the other parameters the same as above, we get $\Gamma_{\perp}^{s}=8.8$ $\times 10^{-17} \mathrm{~kg} \mathrm{~s}^{-1}$ and $\Gamma_{\perp}^{c}=1.2 \times 10^{-13} \mathrm{~kg} \mathrm{~s}^{-1}$ for the spherical and cylindrical tips, respectively. Note that in this case the friction depends on the bias voltage as $V^{4}$.

\section{B. Noncontact friction due to internal friction of the substrate}

In studying the phononic friction in Sec. IV A it was assumed that the deformations of the solids are purely elastic. However, the deformation will be purely elastic or adiabatic only for infinitesimally small velocity, so that at every moment of time the system stays in the equilibrium state. However, real motion always occurs with finite velocity, and the body does not stay in equilibrium. Thus "flow processes" occur, resulting in dissipation of the mechanical energy.

At least two kind of processes result in energy dissipation: (a) heat flow resulting from the temperature gradient, and (b) some kind of internal motion, e.g. point defect flipping. These processes of energy dissipation can be denoted, as in liquids, as internal friction or viscosity.

The friction coefficient due to the internal friction is determined by Eq. (34). However, in contrast to the phononic friction, large values of $q \gg \omega / c_{t}$ play the most important role for the internal friction. For $q \gg \omega / c_{s}$ the tensor component $M_{z z}$ is given by ${ }^{32}$

$$
M_{z z}=\frac{2\left(1-\nu^{2}\right)}{E q},
$$

where $E(\omega)$ is the complex elastic modulus and $\nu$ is the Poisson ratio.

\section{Van der Waals interaction}

For $R \gg d$ only the $\sigma_{z z}$ component of the stress tensor due to the van der Waals interaction is important. In this case, for vibrations of the cylindrical tip parallel to the sample surface, we get

$$
\begin{aligned}
\sigma_{z}(\mathbf{q}) & =\int d^{2} x e^{i \mathbf{q} \mathbf{x}} \frac{\partial}{\partial x} \sigma_{z z}(\mathbf{x}) \\
& =-\frac{i q_{x} R^{1 / 2}}{2^{7} d^{5 / 2}} \frac{\sin \left(q_{y} w / 2\right)}{q_{y}}\left(3+\xi^{2}+3 \xi\right) e^{-\xi},
\end{aligned}
$$

where $\xi=\sqrt{2 d R} q_{x}$. Using (47) and (46) in (34) we get for a cylindrical tip

$$
\Gamma_{\|}^{c}=\frac{75 \pi}{2^{16}} \frac{w \hbar^{2} \omega_{p}^{2}}{d^{6}} \frac{\operatorname{Im}\left(E /\left(1-\nu^{2}\right)\right)}{\omega\left|E /\left(1-\nu^{2}\right)\right|^{2}} .
$$

For the spherical tip similar calculations give

$$
\Gamma_{\|}^{s}=\frac{0.25}{2^{9} \sqrt{2} \pi} \frac{R^{1 / 2} \hbar^{2} \omega_{p}^{2}}{d^{11 / 2}} \frac{\operatorname{Im}\left[E /\left(1-\nu^{2}\right)\right]}{\omega\left|E /\left(1-\nu^{2}\right)\right|^{2}} .
$$

In general, $\operatorname{Im} E(\omega)$ has many resonance peaks, corresponding to different thermally activated relaxation processes. One important source of internal friction at high frequencies is related to thermal currents: elastic compression of a material is commonly associated with heating effects. If the compression takes place sufficiently rapidly, there is no opportunity for heat to be conducted away, while for very slow compression temperature gradients are eliminated by thermal conduction. In both these cases the process of compression will be reversible. In the former case it will be adiabatic and in the latter one, isothermal. In both these limiting cases the contribution from thermal current to the internal friction will be negligible. However, in the intermediate frequency regime we expect dissipation of mechanical energy into heat. The characteristic frequency for the maximum dissipation will be of order $\omega_{t}=1 / \tau$, where, from dimensional arguments, we expect the relaxation time $\tau \sim l^{2} / D$, where $l$ is the linear size of the compression region and $D$ is the thermal diffusibility $D=\kappa / \rho C_{p}$ (where $C_{p}$ is the specific heat and $\kappa$ the heat conductivity). For $l \sim 10^{3} \AA$, this gives for gold $\omega_{t} \approx 10^{11} \mathrm{~s}^{-1}$, which is much higher than the resonance frequency of the cantilever of the atomic force microscope. Another very important contribution to the internal friction is point-defect flipping. This involves thermally activated transitions of point defects or loose sites in crystalline and amorphous networks. A special case is the vibrational motion of adsorbates on the surface of the substrate and/or on the tip, as was treated separately above. Another contribution to the internal friction comes from grain-boundary slip. ${ }^{34}$ For a copper cylindrical tip and a copper substrate, using $d=10 \mathrm{~nm}, w$ $=7 \mu m, R=1 \mu \mathrm{m}, \omega=10^{4} \mathrm{~s}^{-1}$, and, as is typical for metals, ${ }^{35}$ $\operatorname{Im} E(\omega) /|E(\omega)| \approx 10^{-5}$ and $E \approx 10^{11} \mathrm{~N} / \mathrm{m}^{2}$, gives $\Gamma_{\|}^{c}$ $\approx 10^{-16} \mathrm{~kg} \cdot \mathrm{s}^{-1}$. Thus at this separation the internal friction gives much smaller contribution to the friction coefficient than electrostatic friction due to bias voltage or spatial variation of the surface potential. However, internal friction can give the dominant contribution for small separation $d$ $\leqslant 1 \mathrm{~nm}$. For the spherical tip with $R=1 \mu \mathrm{m}$ the friction coefficient is two orders of the magnitude smaller. Finally, we note, as a curiosity, that the internal friction of solids gives a very important contribution to the rolling resistance of most solids, ${ }^{36}$ and is the main contribution to rubber friction on rough substrates, e.g., roadsurface, ${ }^{36}$ where, in the transition 
region between the rubbery and glassy region of the rubber visco-elastic spectra, $\operatorname{Im} E(\omega) /|E(\omega)| \approx 1$.

\section{SUMMARY}

We have studied how the electrostatic friction between an atomic force microscope tip and a substrate depends on (a) the bias voltage, (b) the spatial variation of the surface potential, and (c) the spatial fluctuation of electric charge. We have found that the electrostatic friction can be greatly enhanced in the presence of a 2D system on the surface of the sample or on the tip. On metal surfaces such a $2 \mathrm{D}$ system can result from surface electronic states or from an incommensurate layer of adsorbed ions. We have shown that the experimental data observed in Ref. 3 can be explained by the electrostatic friction in presence of such a $2 \mathrm{D}$ system. The theory predicts the same magnitude, distance, and bias voltage dependence of the friction coefficient as was observed in the experiment, ${ }^{3}$ and explains the bias-voltage-independent contribution to friction. The theory of friction between a gold tip and silica substrate with an inhomogeneous distribution of the charged defects is consistent with the theory of friction between a gold tip and gold substrate. In both theories we have assumed that the gold surfaces are covered by $2 \mathrm{D}$ structure.

The electrostatic friction was compared with the van der Waals friction arising from quantum and thermal fluctuations of the current densities inside the bodies. The van der Waals friction as well as the electrostatic friction can be greatly enhanced in the presence of an identical 2D system on the surfaces of the tip and the substrate. The van der Waals friction is characterized by a stronger distance dependence than the electrostatic friction and may dominate at small separation. The van der Waals friction between 2D systems can be so large that it can be measured with state-of-the-art equipment.

Phonon and internal friction can be ruled out as mechanisms responsible for noncontact friction observed in Ref. 3 because they predict stronger distance and bias voltage dependence. For metal substrate the phonon friction associated with excitation of acoustic phonons is negligibly small in comparison with the electromagnetic friction (especially for motion of the tip parallel to the substrate surface) because of the small area in the phase space available for these phonons.

\section{ACKNOWLEDGMENT}

A.I.V. acknowledges financial support from Russian Foundation for Basic Research (Grant No. 06-02-16979) and ESF "Nanotribology."

\section{APPENDIX A: FRESNEL REFLECTIVITY FOR $P$-POLARIZED ELECTROMAGNETIC WAVES WITH 2D STRUCTURE CORRECTIONS}

We consider a semi-infinite metal having a flat surface that coincides with the $x y$ plane and with the $z$ axis pointed along the inward normal. The metal surface is covered by an adsorbate layer located at $z=-a$. Let the $x z$ plane be the plane of incidence of an evanescent electromagnetic plane wave, with the parallel component of the wave vector $\mathbf{q}$ pointed along the $x$ axis. The macroscopic electric field takes the form

$$
\mathbf{E}=e^{i q x} \times \begin{cases}\mathbf{I} e^{-p z}+\mathbf{R} e^{p z}, & z<-a, \\ \mathbf{A} e^{-p z}+\mathbf{B} e^{p z}, & -a<z<0, \\ \mathbf{T} e^{-s z}, & z>0,\end{cases}
$$

where $p=\left[q^{2}-(\omega / c)^{2}\right]^{1 / 2}, s=\left[q^{2}-(\omega / c)^{2} \varepsilon(\omega)\right]^{1 / 2}$, and $\varepsilon$ is the dielectric function of the metal. According to Ref. 28 the boundary conditions at $z=-a$ can be written in the form

$$
A_{z} e^{p a}+B_{z}^{-p a}-R_{z} e^{-p a}-I_{z} e^{p a}=4 \pi p n_{a} \alpha\left(R_{z} e^{-p a}-I_{z} e^{p a}\right),
$$

$B_{z} e^{-p a}-A_{z} e^{p a}-R_{z} e^{-p a}+I_{z} e^{p a}=-\frac{4 \pi q n_{a} \alpha_{\perp}}{p}\left(R_{z} e^{-p a}+I_{z} e^{p a}\right)$,

where $\alpha_{\|(\perp)}$ is the polarizability of the adsorbate in the direction parallel (normal) to the surface. From the ordinary boundary conditions at $z=0$ it follows

$$
B_{z}=\frac{\varepsilon p-s}{\varepsilon p+s} A_{z} .
$$

For a 2D system $\alpha_{\perp}=0$, and for $q \gg \omega / c$ and $q a \ll 1$ Eqs. (A2)-(A4) give the reflection coefficient $R_{z}$, given by Eq. (18).

\section{APPENDIX B: FRICTION COEFFICIENT DUE TO EXCITATION OF THE ACOUSTIC WAVES}

According to Ref. 32 the tensor $\overleftrightarrow{\mathbf{M}}$ in Eq. (33) is given by

$$
\begin{aligned}
\overleftrightarrow{\mathbf{M}}= & \frac{i}{\rho c_{t}}\left[\frac{1}{S q, \omega}\left[Q(q, \omega)(\hat{z} \mathbf{q}-\mathbf{q} \hat{z})+\left(\frac{\omega}{c_{t}}\right)^{2}\left(p_{l} \hat{z} \hat{z}+p_{t} \hat{q} \hat{q}\right)\right]\right. \\
& \left.+\mathbf{n n} \frac{1}{p_{t}}\right]
\end{aligned}
$$

where $\hat{q}=\mathbf{q} / q, \mathbf{n}=\hat{z} \times \hat{q}$, and

$$
\begin{gathered}
S=\left(\frac{\omega^{2}}{c_{t}^{2}}-2 q^{2}\right)^{2}+4 q^{2} p_{t} p_{l}, \\
Q=2 q^{2}-\omega^{2} / c_{t}^{2}+2 p_{t} p_{l}, \\
p_{t}=\sqrt{\frac{\omega^{2}}{c_{t}^{2}}-q^{2}}, \quad p_{l}=\sqrt{\frac{\omega^{2}}{c_{l}^{2}}-q^{2}} .
\end{gathered}
$$

In the equations above, $\rho, c_{t}$, and $c_{l}$ are the mass density and the transverse and longitudinal sound velocities of the solid, respectively. Note that $c_{t}$ and $c_{l}$ are in general complex frequency-dependent quantities given by

$$
c_{t}^{2}=\frac{E}{2 \rho(1+\nu)},
$$




$$
c_{l}^{2}=\frac{E(1-\nu)}{\rho(1+\nu)(1-2 \nu)},
$$

where $E(\omega)$ is the complex elastic modulus and $\nu$ is the Poisson ratio.

The acoustic waves have wave number $q<\omega / c_{t}$. At typical experimental condition the frequency of the vibrations of the tip $\omega \sim 10^{3}-10^{6} \mathrm{~s}^{-1}$ and $q R_{\text {int }}<\omega R_{\text {int }} / c_{t} \ll 1$, where $R_{\text {int }}$ $\sim \sqrt{d R}$ is the radius of the interaction of the tip with the sample surface. In this case for the vibrations of the tip normal to the surface we get

$$
\begin{aligned}
\sigma_{\perp i}(q) & =\int d^{2} x e^{i \mathbf{q} \mathbf{x}} \frac{\partial}{\partial d} \sigma_{i z}^{0}(\mathbf{x}, d) \approx \delta_{i z} \int d^{2} x \frac{\partial}{\partial d} \sigma_{z z}^{0}(\mathbf{x}, d) \\
& =\frac{\partial}{\partial d} F_{z}(d)
\end{aligned}
$$

where $\sigma_{i z}^{0}$ is the static stress acting on the surface of the sample. Using Eqs. (B7) and (B1) in Eq. (34) we get

$$
\Gamma_{\perp}=\frac{\xi_{\perp}}{4 \pi} \frac{K^{2}}{\rho c_{t}^{3}},
$$

where $\xi_{\perp}=\xi_{\perp l}+\xi_{\perp t}+\xi_{\perp s}, K=\partial F_{z} / \partial d$, and the contributions from the longitudinal $\xi_{\perp l}$, the transverse $\xi_{\perp t}$, and surface (Rayleigh) $\xi_{\perp s}$ acoustic waves are given by

$$
\begin{aligned}
& \xi_{\perp l}=\int_{0}^{c_{t} / c_{l}} d x \frac{\sqrt{\left(c_{t} / c_{l}\right)^{2}-x}}{(1-2 x)^{2}+4 x \sqrt{(1-x)} \sqrt{\left(c_{t} / c_{l}\right)^{2}-x}}, \\
& \xi_{\perp t}=\int_{c_{t} / c_{l}}^{1} d x \frac{4 x\left[x-\left(c_{t} / c_{l}\right)^{2}\right] \sqrt{1-x}}{(1-2 x)^{4}+16 x^{2}\left[x-\left(c_{t} / c_{l}\right)^{2}\right](1-x)},
\end{aligned}
$$

$$
\xi_{\perp s}=\pi \sqrt{x_{c}-\left(c_{t} / c_{l}\right)^{2}} / f^{\prime}\left(x_{c}\right),
$$

where

$$
f(x)=4 x \sqrt{x-1} \sqrt{x-\left(c_{t} / c_{l}\right)^{2}}-(2 x-1)^{2},
$$

and where $x_{c}$ is the solution of the equation $f(x)=0, f^{\prime}$ $=d f(x) / d x$. In Eqs. (B15)-(B17) the sound velocities $c_{t}$ and $c_{l}$ are assumed real, taken at $\omega=0$.

For the vibrations of the tip parallel to the surface the main contribution to the energy dissipation due to excitation of the acoustic waves gives a component of $\sigma_{i}$ which acts in the $z$ direction. For this component we get

$$
\sigma_{\| z}(q)=\int d^{2} x e^{i \mathbf{q} \cdot \mathbf{x}} \frac{\partial}{\partial x} \sigma_{z z}^{0}(\mathbf{x}) \approx i q_{x} F_{z}(d) .
$$

Using Eqs. (B13) and (B1) in Eq. (34) we get

$$
\Gamma=\frac{\xi}{8 \pi} \frac{\omega^{2}}{\rho c_{t}^{5}} F_{z}^{2}(d)
$$

where $\xi_{\|}=\xi_{\| l}+\xi_{\| t}+\xi_{\| s}$

$$
\xi_{\| l}=\int_{0}^{c_{l} / c_{l}} d x x \frac{\sqrt{\left(c_{t} / c_{l}\right)^{2}-x}}{(1-2 x)^{2}+4 x \sqrt{(1-x)} \sqrt{\left(c_{t} / c_{l}\right)^{2}-x}},
$$

$$
\xi_{\perp t}=\int_{c_{t} / c_{l}}^{1} d x x \frac{4 x\left[x-\left(c_{t} / c_{l}\right)^{2}\right] \sqrt{1-x}}{(1-2 x)^{4}+16 x^{2}\left[x-\left(c_{t} / c_{l}\right)^{2}\right](1-x)},
$$

$$
\xi_{\perp s}=\pi x_{c} \sqrt{x_{c}-\left(c_{t} / c_{l}\right)^{2}} / f^{\prime}\left(x_{c}\right),
$$

For most metals $c_{t} / c_{l} \approx 1 / 2$ and for this case $\xi_{\perp}=1.62$ and $\xi_{\|}=1.50$.
${ }^{1}$ I. Dorofeyev, H. Fuchs, G. Wenning, and B. Gotsmann, Phys. Rev. Lett. 83, 2402 (1999).

${ }^{2}$ B. Gotsmann and H. Fuchs, Phys. Rev. Lett. 86, 2597 (2001).

${ }^{3}$ B. C. Stipe, H. J. Mamin, T. D. Stowe, T. W. Kenny, and D. Rugar, Phys. Rev. Lett. 87, 096801 (2001).

${ }^{4}$ H. J. Mamin and D. Rugar, Appl. Phys. Lett. 79, 3358 (2001).

${ }^{5}$ P. M. Hoffmann, S. Jeffery, J. B. Pethica, H. Özgür Özer, and A. Oral, Phys. Rev. Lett. 87, 265502 (2001).

${ }^{6}$ D. Rugar, R. Budakian, H. J. Mamin and B. W. Chui, Nature (London) 430, 329 (2004).

${ }^{7}$ J. A. Sidles, J. L. Carbini, K. J. Bruland, D. Rugar, O. Zuger, S. Hoen, and C. S. Yannoni, Rev. Mod. Phys. 67, 249 (1995).

${ }^{8}$ G. P. Berman, G. D. Doolen, P. C. Hammel, and V. I. Tsifrinovich, Phys. Rev. B 61, 14694 (2000).

${ }^{9}$ N. Arkani-Hamed, S. Dimopoulos, and G. Dvali, Phys. Lett. B 429, 263 (1998); Sci. Am. 283, 62 (2000).

${ }^{10}$ U. Mohideen and A. Roy, Phys. Rev. Lett. 81, 4549 (1998).

${ }^{11}$ T. J. Gramila, J. P. Eisenstein, A. H. MacDonald, L. N. Pfeiffer, and K. W. West, Phys. Rev. Lett. 66, 1216 (1991); Surf. Sci. 263, 446 (1992).
${ }^{12}$ T. J. Gramila, J. P. Eisenstein, A. H. MacDonald, L. N. Pfeiffer, and K. W. West, Phys. Rev. B 47, 12957 (1993); Physica B 197, 442 (1994).

${ }^{13}$ U. Sivan, P. M. Solomon, and H. Shtrikman, Phys. Rev. Lett. 68, 1196 (1992).

${ }^{14}$ I. E. Dzyaloshinskii, E. M. Lifshitz and L. P. Pitaevskii, Adv. Phys. 10, 165 (1961).

${ }^{15}$ A. I. Volokitin and B. N. J. Persson, J. Phys.: Condens. Matter 11, 345 (1999); Phys. Low-Dimens. Struct. 7/8, 17 (1998).

${ }^{16}$ A. I. Volokitin and B. N. J. Persson, Phys. Rev. B 65, 115419 (2002).

${ }^{17}$ B. N. J. Persson and A. I. Volokitin, Phys. Rev. Lett. 84, 3504 (2000).

${ }^{18}$ J. R. Zurita-Sánchez, J. J. Greffet, L. Novotny, Phys. Rev. A 69, 022902 (2004).

${ }^{19}$ A. I. Volokitin and B. N. J. Persson, Phys. Rev. Lett. 91, 106101 (2003).

${ }^{20}$ A. I. Volokitin and B. N. J. Persson, Phys. Rev. B 68, 155420 (2003).

${ }^{21}$ A. A. Chumak, P. W. Milonni, and G. P. Berman, Phys. Rev. B 
70, 085407 (2004).

${ }^{22}$ A. I. Volokitin and B. N. J. Persson, Phys. Rev. Lett. 94, 086104 (2005).

${ }^{23}$ L. D. Landau and E. M. Lifshitz, Electrodynamics of Continuous Media (Pergamon, Oxford, 1960).

${ }^{24}$ C. I. Sukenik, M. G. Boshier, D. Cho, V. Sandoghdar, and E. A. Hinds, Phys. Rev. Lett. 70, 560 (1993).

${ }^{25}$ S. Hudlet, M. S. Jean, C. Guthmann, and J. Berger, Eur. Phys. J. B 2, 5 (1998).

${ }^{26}$ B. N. J. Persson, Phys. Rev. B 44, 3277 (1991).

${ }^{27}$ T. W. Stowe, T. W. Kenny, D. J. Thomson, and D. Rugar, Appl. Phys. Lett. 75, 2785 (1999).
${ }^{28}$ D. C. Langreth, Phys. Rev. B 39, 10020 (1989).

${ }^{29}$ P. Senet, J. P. Toennis, and G. Witte, Chin. Phys. Lasers 299, 389 (1999).

${ }^{30}$ U. Hartmann, Phys. Rev. B 42, 1541 (1990); 43, 2404 (1991).

${ }^{31}$ P. Johansson and P. Apell, Phys. Rev. B 56, 4159 (1997).

${ }^{32}$ B. N. J. Persson, J. Chem. Phys. 115, 3840 (2001).

${ }^{33}$ B. N. J. Persson and R. Ryberg, Phys. Rev. B 32, 3586 (1985).

${ }^{34}$ F. A. McClintock and A. S. Argon, Mechanical behavior of material (Adison-Wesley, Reading, MA, 1966), pp. 475-483.

${ }^{35}$ B. N. J. Persson, Sliding friction: Physical Principle and Applications, 2nd ed. (Springer, Heidelberg, 2000).

${ }^{36}$ B. N. J. Persson, Surf. Sci. 401, 445 (1998). 